\title{
Formación del Nervio Sural en Individuos Chilenos
}

\author{
Sural Nerve Formation in Chilean Individuals
}

"Olave, E.; "** Cruzat, C.; **** Retamal, P. \& ***Galaz, C.

OLAVE, E.; CRUZAT, C.; RETAMAL, P. \& GALAZ, C. Formación del nervio sural en individuos chilenos. Int. J. Morphol., 28(1):273-276, 2010.

RESUMEN: La inervación cutánea de la región posterior de la pierna esta dada por el nervio cutáneo sural medial (NCSM), el nervio cutáneo sural lateral (NCSL), el nervio sural (NS) y el nervio safeno. Estos ramos son variables pudiendo los dos primeros, ser independientes o estar unidos y formar el nervio sural. Con el propósito de determinar la formación del nervio sural y los niveles de origen de los nervios NCSM y NCSDL, disecamos 12 piernas formolizadas, 7 izquierdas y 5 derechas, de individuos chilenos, adultos. El punto de origen y unión de los nervios NCSM y NCSL se localizó en relación a una línea transversal trazada entre los puntos más prominentes de los cóndilos femorales (LBE), así como una línea trazada entre los dos maléolos (LBM). La distancia entre ambas líneas fue en promedio $31,8 \mathrm{~cm}$; el punto de origen del NCSL se situó en promedio a 3,75 cm proximal a la LBE y el del NCSM a 2,23 cm proximal a la misma línea. En 7 muestras $(58,3 \%)$ observamos unión de los dos ramos formando el NS ( en dos de ellas a través de un ramo comunicante fibular) y en las restantes se mantuvieron independientes. El punto de unión de los nervios referidos fue localizado proximal a la LBM. Los resultados obtenidos complementarán el conocimiento anatómico de la inervación sensitiva de la pierna.

PALABRAS CLAVE: Anatomía; Inervación; Pierna; Nervio Sural.

\section{INTRODUCCIÓN}

La anatomía de la inervación sensitiva de la región posterior de la pierna es compleja, debido a que los nervios involucrados pueden estar unidos o ser independientes, por lo que es necesario tener un conocimiento acabado de su origen, disposición y distribución, contribuyendo con esto a la anatomía quirúrgica de la región. Textos clásicos de Anatomía no se refieren con detalle a estos nervios, entre los cuales se puede encontrar al nervio sural, descrito de manera muy general por Williams et al. (1995), nervio cutáneo sural medial, nervio cutáneo sural lateral y ramo comunicante fibular.

Según Dangelo \& Fattini (1988) antes de la división del nervio fibular común, éste emite un nervio cutáneo, el cutáneo sural lateral, que inerva la piel de la región lateral y posterior de la pierna, el que a su vez, emite el ramo comunicante fibular, el cual se une al nervio cutáneo sural medial, ramo del nervio tibial, formando de este modo al nervio sural, cuya disposición es la más frecuente, lo cual no excluye posibles variaciones.

En una investigación realizada por Ortigüela et al. (1987) se encontró que en la mayoría de los 20 casos estu- diados $(80 \%)$ el nervio sural se formó en la porción distal de la pierna por la unión del nervio cutáneo sural medial y el ramo comunicante fibular.

Por su parte, Drizenko et al. (2004) estudiaron 55 miembros inferiores, encontrando que en $67 \%$ de ellos el nervio sural se formó por los nervios cutáneos sural medial y lateral, uniendo a ambos, el ramo comunicante fibular. La formación del nervio sural a partir del nervio cutáneo sural medial se presentó en $29 \%$ y en sólo 3,6 \% el nervio cutáneo sural lateral fue responsable de su formación.

En otra serie de muestras (30), Aktan et al. (2005) señalaron que en $60 \%$ de los casos, el nervio sural se formó por la unión de los nervios cutáneos sural medial y lateral, a nivel de los dos tercios superiores de la pierna.

Recientemente, Apaydin et al. (2009) señalaron que el nervio sural se presentó bilateralmente en todos los casos que estudiaron (44). Estos autores indicaron también que durante la reparación percutánea del tendón calcáneo, el nervio sural tiene un alto riesgo de ser lesionado.

\footnotetext{
* Facultad de Medicina, Universidad de La Frontera, Temuco, Chile.

** Facultad de Medicina, Universidad Católica del Maule,Talca, Chile.

*** Alumnos Carrera de Medicina, Facultad de Medicina, Universidad Católica del Maule,Talca, Chile.
} 
Basado en lo anterior, realizamos un estudio con el objetivo de verificar la presencia del nervio sural y de los ramos involucrados en su formación, determinar desde el punto de vista biométrico el punto de origen de sus componentes y el punto de unión de los nervios formadores del nervio sural.

\section{MATERIAL Y MÉTODO}

El estudio se llevó a cabo en 12 miembros inferiores (7 izquierdos y 5 derechos) de individuos Chilenos, adultos, fijados en formaldehído al $10 \%$, localizados en los Laboratorios de Anatomía de la Facultad de Ciencias de la Salud, Universidad Católica del Maule, Talca, Chile.

Cada uno de los casos fue disecado, incidiendo la piel de la región posterior de la pierna, a nivel de la línea media, reclinándola hacia ambos lados. Luego se procedió a identificar a los nervios cutáneos posteriores y así determinar la presencia del nervio sural y su formación.

Para el registro biométrico del origen de los nervios cutáneos sural medial y lateral, así como el del ramo comunicante fibular y punto de unión de los mismos, se consideró como punto de referencia una línea trazada entre las partes más prominentes de los cóndilos femorales (LBE) y otra entre los maléolos medial y lateral (LBM), distancia que se dividió en tercios para una mejor localización del punto de unión cuando lo hubo. Para el registro de medidas de utilizó una huincha métrica y un caliper digital.

Todas las muestras fueron esquematizadas y se obtuvieron fotografías de las más representativas.

\section{RESULTADOS}

La distancia entre las líneas LBE y LBM fue en promedio $31,8 \mathrm{~cm}$., con rangos entre 29,0 y $34,0 \mathrm{~cm}$; el punto de origen del nervio cutáneo sural lateral se situó en promedio a $3,75 \mathrm{~cm}$ proximal a la LBE con rangos entre 1,0 y $7,0 \mathrm{~cm}$ y el del nervio cutáneo sural medial a $2,23 \mathrm{~cm}$ proximal a la misma línea, con rangos entre 0,0 y $4,5 \mathrm{~cm}$.

En 7 muestras $(58,3 \%)$ observamos la unión de los dos ramos mencionados previamente formando el nervio sural (Figs.1 y 3), de las cuales, dos de ellas (16,7 \% del total estudiado) presentaron dicha unión a través del ramo comunicante sural y en las restantes muestras $(41,7 \%)$ se mantuvieron independientes (Fig. 2). En los casos que hubo unión de ramos para formar el nervio sural, el punto de unión fue localizado proximal a la $\operatorname{LBM}(7,5 ; 13,5 ; 3,5 ; 15,0$ y $7,0 \mathrm{~cm})$; en los dos casos en que se presentó el ramo comunicante sural, la unión

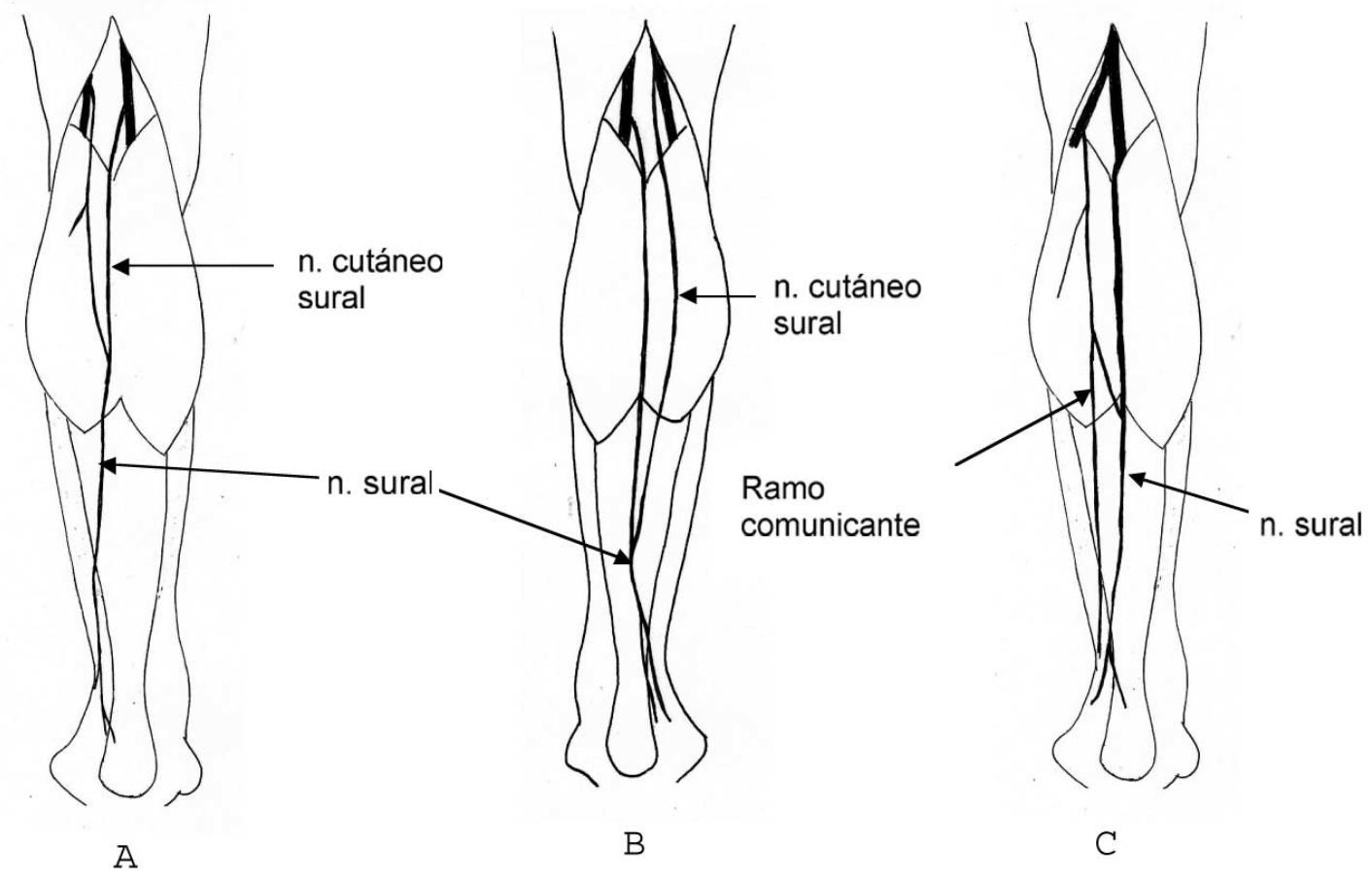

Fig. 1. Tipos de unión de los nervios cutáneos sural lateral y sural medial para formar al nervio sural. 
de éste con el nervio cutáneo sural medial se realizó a $15,3 \mathrm{~cm}$ y $11,7 \mathrm{~cm}$ proximal a la LBM, respectivamente. El punto de unión se localizó en el tercio medio de la distancia LBE-LBM en 4 casos y en el tercio distal en los 3 casos restantes.

En los casos que no se unieron los nervios cutáneos sural medial y lateral, el primero de ellos descendió como nervio sural.

\section{DISCUSIÓN}

La inervación cutánea en las diferentes partes del cuerpo es de vital importancia por causa de las sensaciones externas que debemos atender y procesar para su debida respuesta. De tal modo que, se debe poner atención especial a los nervios involucrados en ello, conociendo su localización, disposición, distribución y variaciones en las áreas correspondientes.

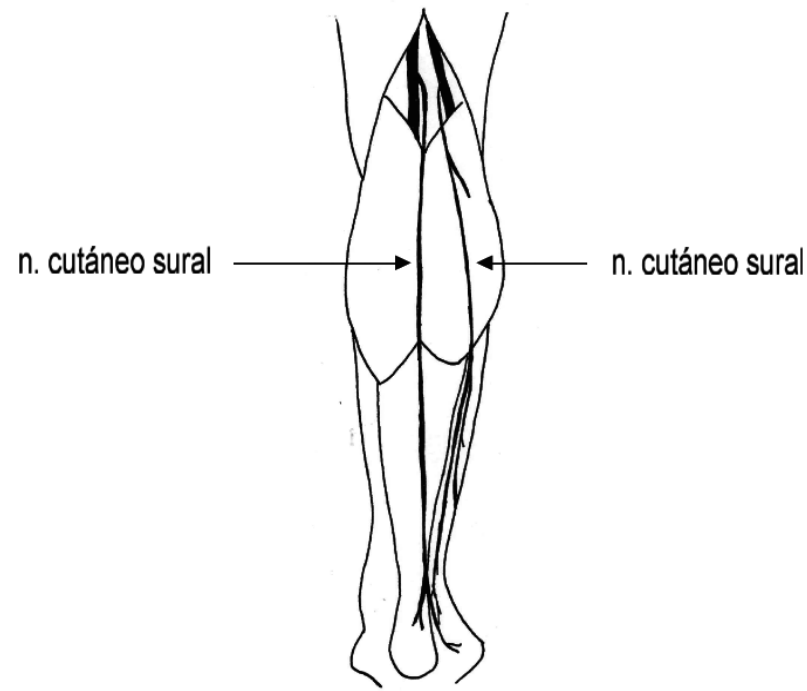

Fig. 2. Disposición de los nervios cutáneos sural lateral y medial, de trayecto independiente.
Los nervios relacionados a la región posterior de la pierna tienen relaciones importantes con el tendón calcáneo y durante la reparación percutánea de éste, el nervio sural tiene un alto riesgo de ser lesionado (Apaydin et al.).

La literatura clásica describe que en la región aludida se disponen los nervios cutáneo sural lateral, cutáneo sural medial, ramo comunicante sural, sural y safeno (Testut \& Latarjet, 1969; Healey \& Seybold, 1972; Williams et al.; Moore \& Dalley, 2002).

Estos nervios se presentan independientes o la unión de algunos dar origen al nervio sural, dejando en claro que el nervio safeno es responsable de una pequeña área de inervación en esta región, siendo además de origen alto y procediendo del nervio femoral, ramo del plexo lumbar.

Descripciones específicas muestran porcentajes de presencia independiente o nervios derivados de la unión de algunos. Así, Ortigüela et al. encontraron que en el $80 \%$ de su serie (20 casos), el nervio sural se formó en la porción distal de la pierna por la unión del nervio cutáneo sural medial y el ramo comunicante fibular. En $20 \%$ de las muestras, este último estuvo ausente y en esos casos, el nervio sural derivó directamente del nervio cutáneo sural medial.

Por su parte, Drizenko et al. estudiaron 55 miembros inferiores, encontrando que en $67 \%$ de ellos, el nervio sural se formó por los nervios cutáneos sural medial y lateral, uniendo a ambos, el ramo comunicante fibular. Nuestros resultados son concordantes con los de estos autores, respecto a la unión de los dos nervios aludidos para formar el nervio sural, sin embargo, la unión a través del ramo comunicante estuvo presente en nuestro grupo de estudio sólo en 16,7\%. La formación del nervio sural a partir del nervio cutáneo sural medial que los autores mencionados observaron en $29 \%$, estuvo presente en todos los casos en que no hubo unión en nuestra serie

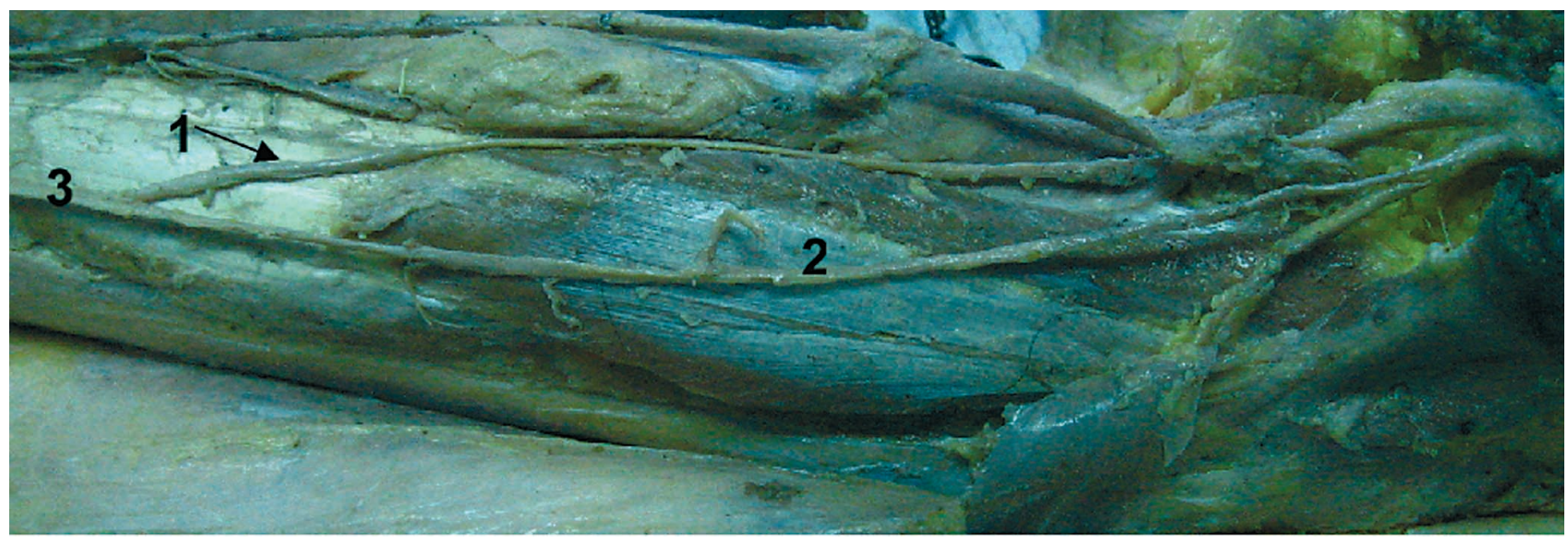

Fig. 3. Los nervios cutáneos sural medial (1) y sural lateral (2) forman al nervio sural (3) a nivel del tercio distal de la distancia LBE-LBM. 
$(41,7 \%)$, porcentaje superior al mostrado en su investigación, que creemos podría ser por la diferencia en el número de muestras.

Aktan et al. observaron que en $60 \%$ de los casos (30 en total), el nervio sural se formó por la unión de los nervios cutáneos sural medial y lateral, a nivel de los dos tercios superiores de la pierna, resultado diferente del obtenido en nuestra serie donde esa unión, cuando existe, la observamos con mayor frecuencia a nivel de los dos tercios inferiores. Estos autores observaron la unión de los nervios a nivel distal en sólo un $10 \%$ y señalaron que en el 6,7 \% los nervios tuvieron trayectos separados, estando ausente el nervio cutáneo sural lateral en 16,7 \% y el medial en 6,7 \% de los casos.

En general, se puede señalar que los nervios cutáneos sural medial y lateral están siempre presentes, uniéndose en poco más de la mitad de los casos para formar al nervio sural. En cuanto al origen de los nervios mencionados, siempre es proximal a la línea biepicondilar. En los casos de unión de ambos ramos o unión a través del ramo comunicante fibular, el punto de unión puede localizarse en el tercio medio o distal de la distancia LBE-LBM.

Cuando no hay unión de los nervios cutáneos sural medial y lateral, el primero de ellos tiene el trayecto y la distribución del nervio sural.

Los datos obtenidos en este trabajo son un aporte al conocimiento de la anatomía de los nervios cutáneos de nuestra población, pudiendo ser de utilidad a los procedimientos quirúrgicos que se efectúan en la región.

OLAVE, E.; CRUZAT, C.; RETAMAL, P. \& GALAZ, C. Sural nerve formation in Chilean individuals. Int. J. Morphol., 28(1):273-276, 2010.

SUMMARY: The skin of the posterior region of the leg is supplied by the medial sural cutaneous (MSCN), lateral sural cutaneous (LSCN), sural (SN) and the safenous nerves. These nerves present variations where the first and second nerves can be independents or both join to form the sural nerve. The objective of this research was to determine the sural nerve formation and the origin level of the MSCN and LSCN. We dissected 12 legs fixed in formaldehyde 10\% ( 7 of the left side and 5 of the right side), from of adult Chilean individuals. The origin and join points of the MSCN and LSCN nerves were localized in relation to horizontal line between the prominent points of the femoral condyles (BCL), so as a horizontal line between the two malleolus (BML), respectively. The distance between both lines was average $31.8 \mathrm{~cm}$; the origin point of the LSCN was $3.75 \mathrm{~cm}$ proximal to BCL and the origin point of the MSCN was $2.23 \mathrm{~cm}$ proximal to this line. In 7 cases we observed both nerves forming the sural nerve (in two of them, by a fibular communicant branch) and in rest cases were independents. The joint point of the referred nerves was localized proximal to BML. These results are a contribution to the anatomic knowledge of the sensitive innervation of the leg.

KEY WORDS: Anatomy; Innervation; Leg; Sural nerve.

\section{REFERENCIAS BIBLIOGRÁFICAS}

Aktan Ikiz, Z.A.; Uçerler, H. \& Bilge, O. The anatomic features of the sural nerve with an emphasis on its clinical importance. Foot Ankle Int., 26(7):560-7, 2005.

Apaydin, N.; Bozkurt, M.; Loukas, M.; Vefali, H.; ShaneTubbs, R. \& Esmer, F. Relationships of the sural nerve with the calcaneal tendon: an anatomical study with surgical and clinical implications. Surg. Radiol. Anat., 2009.

Dangelo, J. \& Fattini, C. Anatomia Humana Sistêmica y Segmentar. 2a Ed. Rio de Janeiro, Atheneu, 1988.

Drizenko, A.; Demondion, X.; Luyckx, F.; Mestdagh, H. \& Cassagnaud, $\mathrm{X}$. The communicating branches between the sural and superficial peroneal nerves in the foot: a review of 55 cases. Surg. Radiol. Anat., 26:447-452, 2004.

Healey, J. \& Seybold, W. Anatomía Clínica. $1^{a}$ ed. México, NuevaInteramericana, 1972.
Moore K.L. \& Dalley A.F. Anatomía con Orientación Clínica. $4^{a}$ Ed. Panamericana, Buenos Aires, 2002.

Ortigüela, M. E.; Wood, M. B. \& Cahill; D. R. Anatomy of the sural nerve complex. J. Hand Surg. Am., 12(6):1129-23, 1987.

Testut L, Latarjet A. Tratado de Anatomía Humana. Salvat, Barcelona, 1969.

Williams, P. L; Warwick, R.; Dyson, M. \& Bannister, LH. Gray Anatomia. $37^{\text {th }}$ Ed. Rio de Janeiro, Guanabara Koogan, 1995.

Dirección para correspondencia:

Prof. Dr. Enrique Olave

Facultad de Medicina

Universidad de La Frontera

Casilla 54-D

Temuco - CHILE

Email: eolave@ufro.cl
Recibido : 11-11-2009

Aceptado: 18-01-2010 